Internat. J. Math. \& Math. Sci.

Vol. 24, No. 1 (2000) 5-9

S0161171200002659

(c) Hindawi Publishing Corp.

\title{
THE FOURIER TRANSFORMS OF LIPSCHITZ FUNCTIONS ON THE HEISENBERG GROUP
}

\author{
M. S. YOUNIS
}

(Received 1 December 1998)

\begin{abstract}
We study the order of magnitude of the Fourier transforms of certain Lipschitz functions on the Heisenberg group $H^{n}$. We compare our conclusions with some previous results in the field.
\end{abstract}

Keywords and phrases. Fourier transforms, absolute convergence, Lipschitz functions, Heisenberg group.

2000 Mathematics Subject Classification. Primary 42A38, 44A15; Secondary 42C99, 44A05.

1. Introduction. In [2, Theorem 2.3], Inglis proved for Lipschitz functions on the Heisenberg group an analogue of Bernstein's theorem on the absolute convergence of the Fourier series of Lipschitz functions of order $\alpha>1 / 2$ on the circle group $T=$ $[1,2 \pi]$. Pini also proved in [3] a similar theorem for Lipschitz functions on SU(2) the special unitary group of matrices of order 2 . In this paper, we prove some results on the order of magnitude of the Fourier transforms of some Lipschitz classes on $H^{n}$, comparing them with those obtained in [2, 3].

DEFinitions AND NOTATION. We assume that the reader is familiar with the group-theoretic Fourier transform as can be found in [1] for instance. The Heisenberg group $H^{n}$ is the $2 n+1$-dimensional nilpotent Lie group with its underlying manifold $R \times C^{n}=R^{2 n+1}, R$ and $C$ being real and complex Euclidean spaces, respectively. An element $g$ in $H^{n}$ is written as $g=\left(p, q, t 1, p, q \in R^{n}, t \in R\right)$. The dot product $p \cdot q=p_{1} q_{1}+p_{2} q_{2}+\cdots+p_{n} q_{n}$ is frequently used. The first difference with step $h_{j}$ in $x_{j}$ is given by

$$
\Delta_{h_{j}} f(x)=f\left(\ldots, x_{j}+h_{j}, \ldots\right)-f\left(\ldots, x_{j}, \ldots\right),
$$

where $x=\left(x_{1}, x_{2}, \ldots, x_{n}\right), h=\left(h_{1}, h_{2}, \ldots, h_{2 n+1}\right)$. The symbol $\Delta_{h_{j} h_{j}} f(x)$ stands for $\Delta_{h_{j}}\left(\Delta_{h_{i}} f(x)\right)$, the $n$th difference $\Delta_{h}^{n} f(x)$ is defined inductively. On a locally compact group $G$ with its dual object $\hat{G}$ the Fourier transform $\hat{f}$ of a function $f(g)$ in $L^{1}(G)$ is defined by

$$
\hat{f}(\pi)=\int_{G} f(g) \pi\left(g^{-1}\right) d g, \quad g \in G,
$$

where $\pi$ is the irreducible unitary representation on $G$. A suitable form of $\pi$ on $H^{n}$ is the following:

$$
\pi(p, q, t) u(x)=e^{-i \lambda(t+q \cdot x+p \cdot q / 2)} u(x+P),
$$


$u \in L^{2}\left(R^{n}\right), x, p, q \in R^{n}, \lambda \in R \backslash 0$ (see [5, page 49]). The Haar measure on $H^{n}$ is the Lebesgue measure on $R^{2 n+1}$. An increment in $g \in H^{n}$ is given by $h=\left(h_{1}, h_{2}, \ldots, h_{2 n+1}\right)$ in the $2 n+1$-variables $p, q$, and $t$, where $|h|=\sum_{j=1}^{2 n+1}\left|h_{j}\right|^{2}$. Since $h_{j}=0(|h|)$ for $j=1,2, \ldots, 2 n+1$, we take $h_{1}, h_{2}, \ldots, h_{2 n+1}$ to be equal to $h$. This will simplify the proof considerably. We introduce the following.

DeFinITION 1.1. Let $f \in L^{p}\left(H^{n}\right)$. Then $f$ is said to belong to the Lipschitz class Lip $(\alpha, p), \alpha>0$ if

$$
\left\|\Delta_{h}^{2 n+1} f\right\|_{p}=0\left(|h|^{\alpha}\right), \quad h \longrightarrow 0,
$$

where $\|\cdot\|_{p}$ is the usual $L^{p}$-norm on $H^{n}$.

2. Main theorems. Our main theorem is stated as follows.

THEOREM 2.1. Let $f \in L^{P}\left(H^{n}\right), 1<P \leq 2$, such that (1.4) is satisfied. Then $\|\hat{f}\|_{\mathrm{HS}}^{r}$ belongs to $L^{1}(0, \infty)$ for

$$
\frac{(2 n+1) P}{\alpha P+(3 n+1) P-(3 n+1)}<r \leq q=\frac{P}{P-1},
$$

where $\|\hat{f}\|_{\mathrm{HS}}$ is the Hilbert-Schmidt norm of $\hat{f}$.

Proof. The Fourier transform of $f$ is given by

$$
\hat{f}(\lambda, x)=\int_{H^{n}} f(p, q, t) e^{-i \lambda(t+q \cdot x+p \cdot q / 2)} d p d q d t .
$$

We mention that in some definitions of $\hat{f}$ (cf. [5, page 49]) the exponents in (2.2) take different signs \pm . This has no bearing on our proof, since we are dealing with the absolute value of the exponential function. Turning now to the transform of $\Delta_{h}^{2 n+1} f$, we see that the difference in step $h$ with respect to $t$ yields the factor $\left(e^{-i \lambda h}-1\right)$, the $n$th difference in $q$ for $q \cdot x$ gives the factor $\prod_{j=1}^{n}\left(e^{-i \lambda h x_{j}}-1\right)$. Since this product depends on $x$, it will be included in the Hilbert-Schmidt-norm of $\hat{f}$ without changing the conclusion of the theorem. Finally, the difference resulting from $p \cdot q / 2$ yields the product

$$
\prod_{j=1}^{n}\left[\left(e^{-i \lambda h q_{j}}-1\right)\left(e^{-i \lambda h p_{j}}-1\right)\right] .
$$

This quantity depends on $p, q$, therefore it is embraced in the integral defining $\hat{f}$. Apart from a bounded multiplicative constant $\left(2^{2 n}\right)$ it has no major role in the proof. Thus one finally arrives at the following:

$$
\left|\Delta_{h}^{2 n+1} f\right|=0\left|\left[\left(e^{-i \lambda h}-1\right) \prod_{j=1}^{n}\left(e^{-i \lambda h x_{j}}-1\right)\right] \hat{f}(\lambda, x)\right| .
$$

The Housdorfl Young inequality yields

$$
\begin{aligned}
\int_{0}^{\infty}|\sin \lambda h|^{q}\left\|\prod_{j=1}^{n}\left|\sin \lambda h x_{j}\right| \hat{f}(\lambda, x)\right\|_{\mathrm{HS}}^{q}|\lambda|^{n} d \lambda & =\int_{0}^{\infty}|\sin \lambda h|^{q}\|\hat{f}(\lambda)\|_{\mathrm{HS}}^{q}|\lambda|^{n} d \lambda \\
& \leq A\|f\|_{p}^{q}=0\left(|h|^{\alpha q}\right) .
\end{aligned}
$$


Since $|\sin \lambda h| \geq A|\lambda h|$ for $0<\lambda<1 / h, A$ being constant, hence

$$
\begin{aligned}
& \int_{0}^{1 / h}|\lambda h|^{q}\|\hat{f}\|_{\mathrm{HS}}^{q}|\lambda|^{n} d \lambda=0\left(|h|^{\alpha q}\right), \\
& \int_{0}^{1 / h}|\lambda|^{n+q}\|\hat{f}\|_{\mathrm{HS}}^{q} d \lambda=0\left(|h|^{\alpha q-q}\right) .
\end{aligned}
$$

For $\lambda \geq 1$, we introduce the function

$$
\Phi(X)=\int_{1}^{X}|\lambda|^{n+q}\|\hat{f}\|_{\mathrm{HS}}^{q} d \lambda .
$$

Then $\|\hat{f}\|_{\mathrm{HS}}^{q}=0|\lambda|^{-n-q}(d \Phi / d \lambda)$, so that

$$
\int_{1}^{X}\|\hat{f}\|_{\mathrm{HS}}^{q} d \lambda=0\left(X^{-n-q} \Phi(X)\right)=0\left(X^{-n-\alpha q}\right)
$$

plus terms of the same order. For $r \leq q$ the Hölder's inequality applied to the last quantity yields

$$
\begin{aligned}
\int_{1}^{X}\|\hat{f}\|_{\mathrm{HS}}^{r} d \lambda & =0\left(X^{(-n-\alpha q) r / q+(2 n+1)-(2 n+1) r / q}\right) \\
& =0\left(X^{(2 n+1)-\alpha r-(3 n+1) r+(3 n+1) r / p}\right),
\end{aligned}
$$

giving the required condition for the boundedness of this estimate for large $X$. This completes the proof.

In view of the complete symmetry of the $L^{2}$ theory of the Fourier transform, one can formulate Theorem 2.1 as follows.

THEOREM 2.2. Let $f$ belong to $L^{2}\left(H^{n}\right)$. Then the conditions

$$
\begin{gathered}
\left\|\Delta_{h}^{2 n+1} f\right\|_{2}=0\left(|h|^{\alpha}\right), \quad \alpha>0, h \rightarrow 0, \\
\int_{X}^{\infty}|\lambda|^{n}\|\hat{f}\|_{\mathrm{HS}}^{2} d \lambda=0\left(X^{-2 \alpha}\right), \quad \int_{X}^{\infty}\|\hat{f}\|_{\mathrm{HS}}^{2} d \lambda=0\left(X^{-n-2 \alpha}\right),
\end{gathered}
$$

as $X \rightarrow \infty$ are equivalent. The method of the proof is explained in [6, page 117] and $[7,8]$, and will not be given here. Of particular interest is the special case $P=2, r=1$ in (2.6) which yields one form of the absolute convergence of the Fourier transform on $H^{n}$. Namely one obtains in this case

$$
\int_{1}^{X}\|\hat{f}\|_{\mathrm{HS}} d \lambda=0\left(X^{(n+1) / 2-\alpha}\right),
$$

which is bounded for large $X$ if $\alpha>(n+1) / 2$. This result is perhaps the nearest analogue for Lipschitz function on $H^{n}$ to the Bernstein's theorem.

2.1. Concluding remarks. We point out first in [3] the subscripts 2,1 are not properly placed in the definition of the Besov space which is usually written as $\Delta_{2,1}^{\alpha}$ rather than as $\Delta_{2,2}^{\alpha}$. We also add that the relation between the smoothness exponent $\alpha$ and the dimension of $\mathrm{SU}(2)(\alpha>3 / 2)$ in [3] is more indicative than the corresponding 
relation $\alpha>1 / 2$ in [2]. Comparing the present method with that followed in [2, 3] one can say that inspite of its elegance, the proof there conceals many concrete cases and important aspects that should be more salient in the issue under discussion. The role of the Fourier transform (coefficients) as well as the effect of Lipschitz conditions on $\hat{f}$ are hardly sensed in that proof, had it not been for the inclusion of $\alpha$ in the definition of $\Delta_{2,1}^{\alpha}$. In contrast, the present method provides a variety of estimates, giving clear relations between $\alpha, n, p$, and $r$. This makes our method more applicable in other areas such as approximation theory and weighted norm inequalities (for example) which are vital topics on their own. Another point of interest is that the condition $\alpha>1 / 2$ given in [2] is rather vague. (Nearly all the papers written on this subject relate $\alpha$ to the group dimension as well as to the space exponent.) We think that this relation is partly concealed in the metric structure $(d t, z)=\left(t^{2}+1 z 1^{4}\right)^{(n+1) / 2}$ of $H^{n}$, and thatpartly-it is tacitly included in the condition $k=n+2$, which together with $\alpha=1 / 2$ provides the smoothness condition for $\Delta_{2,1}^{\alpha}$. It would be quite relevant to mention here that there are several criteria for the absolute convergence of the Fourier transforms (coefficients) on non-Abelian groups (cf. [4]). This may explains-partially-the variation in the range of $\alpha$ mentioned earlier.

Our final comment is rather a heurisatic comparison between harmonic analysis on $H^{n}$ and $R^{2 n+1}$. Since $H^{n}$ is nearly Euclidean in its structure, then it is quite natural that analysis on $H^{n}$ has something in common with that carried on $R^{2 n+1}$ and that the Fourier transform (in particular) on $H^{n}$ inherits some properties of the transform on $R^{2 n+1}$. This must have been felt from the above analysis. For example, Taylor (see [5, page 52]), views $\hat{f}$ for $f \in L^{2}\left(H^{n}\right)$ as a Fourier transform $\hat{k}(\lambda, y, x)$ of three parametric variables (i.e., $2 n+1$ variables) whereas in view of the structure of the representation on $H^{n} \hat{f}=\hat{k}$ is in fact a function of $\lambda$ and $x$ only $(n+1$ variables). Thus in our situation the effective dimension of $H^{n}$ (roughly speaking) is $n+1$ rather than $2 n+1$ (in that case $\alpha$ ) should be greater than $(2 n+1) / 2$ for the absolute convergence of $\hat{k}$ if $k(p, q, t)$ belongs to $L^{2}\left(R^{2 n+1}\right)$. This explains the occurrence of the weight $|\lambda|^{n}$ in the Parseval's identity for $H^{n}$ (the Plancherel's measure $=|\lambda|^{n} d \lambda$ ). Had $\hat{f}$ been of the form $\hat{f}(\lambda, y, x)$, then this would have definitely influenced both $\|\hat{f}\|_{\mathrm{HS}}^{2}$ and the Plancherel's measure. The presence of $|\lambda|^{n}$ in the Parseval's identity enhances the rapidity of convergence of $\|\hat{f}\|_{\mathrm{HS}}^{2}$ and hence affects the order of magnitude of other quantities depending on it as might have been noticed. In our view, the main reason is that the factor $e^{-i \lambda p \cdot q / 2}$ in $\pi(p, q, t)$ is ineffective in connection with the smoothness conditions and that it curtails the dimension of $H^{n}$. Taking this into consideration and bearing in mind the metric structure of $H^{n}$ one could see why $\alpha=1 / 2$ in Inglis's theorem is sufficient for the membership of $\Delta_{2,1}^{\alpha}$ in $A\left(H^{n}\right)$, (the Fourier algebra of $H^{n}$ ).

\section{REFERENCES}

[1] E. Hewitt and K. A. Ross, Abstract Harmonic Analysis. Vol. II: Structure and Analysis for Compact Groups. Analysis on Locally Compact Abelian Groups, Springer-Verlag, New York, 1970. MR 41\#7378. Zbl 213.40103.

[2] I. R. Inglis, Bernstein's theorem and the Fourier algebra of the Heisenberg group, Boll. Un. Mat. Ital. A (6) 2 (1983), no. 1, 39-46. MR 84g:43014. Zbl 528.43008.

[3] R. Pini, Bernstein's theorem on SU(2), Boll. Un. Mat. Ital. A (6) 4 (1985), no. 3, 381-389. MR 87k:43010a. Zbl 612.43010. 
[4] D. L. Ragozin, Approximation theory, absolute convergence, and smoothness of random Fourier series on compact Lie groups, Math. Ann. 219 (1976), no. 1, 1-11. MR 53\#13961. Zbl 307.43012.

[5] M. E. Taylor, Noncommutative Harmonic Analysis, Mathematical Surveys and Monographs, 22, American Mathematical Society, Providence, R.I., 1986. MR 88a:22021. Zbl 604.43001.

[6] E. C. Titchmarsh, Theory of Fourier Integrals, 2nd ed., Oxford University Press, 1948.

[7] M. S. Younis, Fourier transforms of Dini-Lipschitz functions, Internat. J. Math. Math. Sci. 9 (1986), no. 2, 301-312. MR 88b:42019. Zbl 595.42006.

[8] _ Fourier transformations of functions with symmetrical differences, Acta Math. Hungar. 51 (1988), no. 3-4, 293-299. MR 89k:42009. Zbl 672.42005.

M. S. YOUNIS: DEPARTMENT OF MATHEMATICS, YARMOUK UNIVERSITY, IRBID, JORDAN 


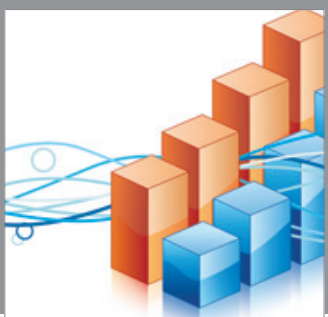

Advances in

Operations Research

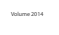

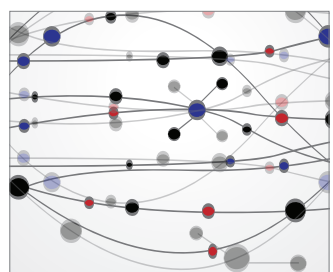

\section{The Scientific} World Journal
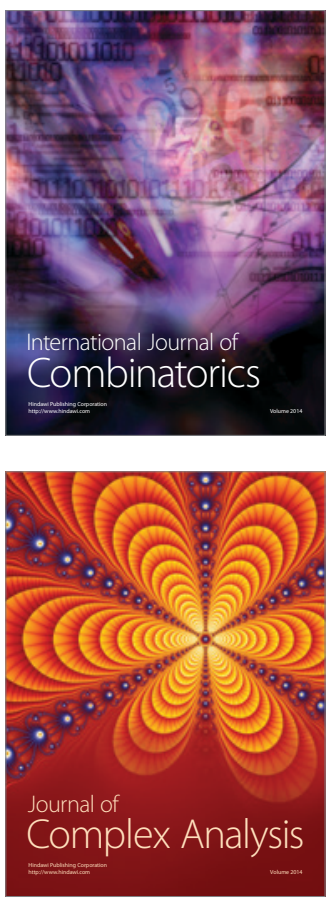

International Journal of

Mathematics and

Mathematical

Sciences
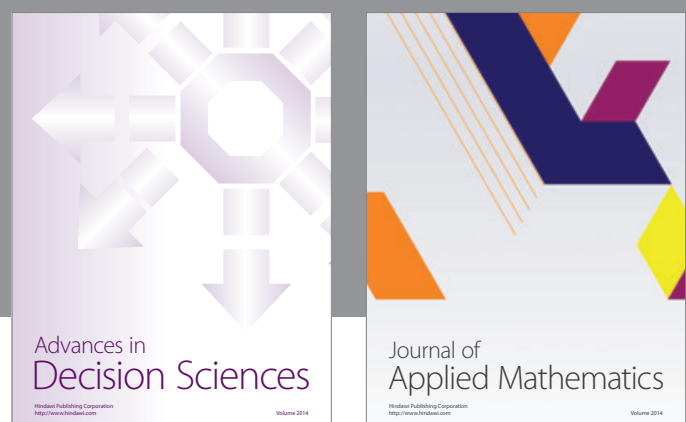

Journal of

Applied Mathematics
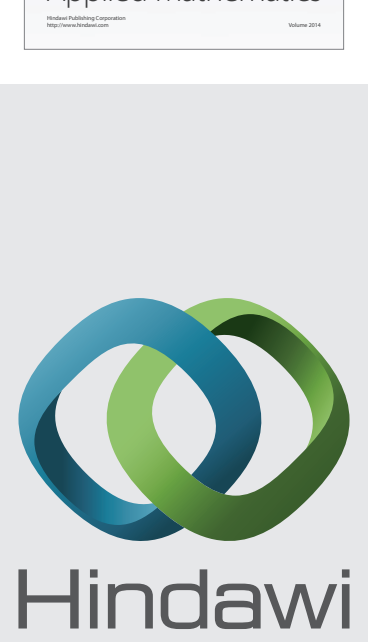

Submit your manuscripts at http://www.hindawi.com
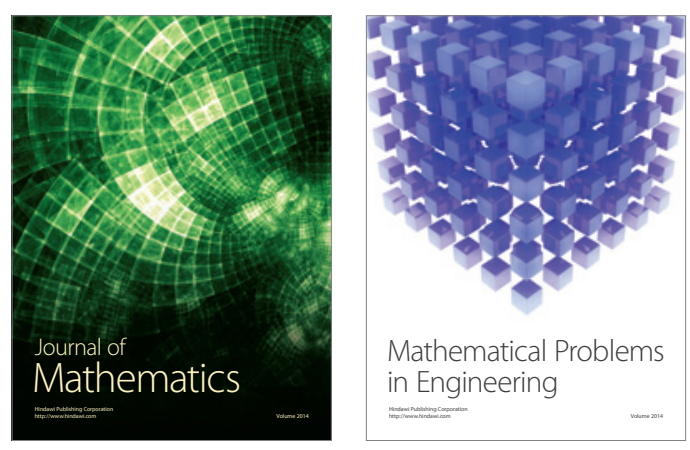

Mathematical Problems in Engineering
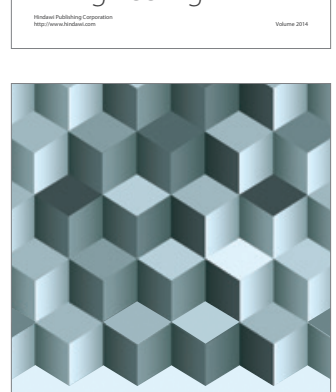

Journal of

Function Spaces
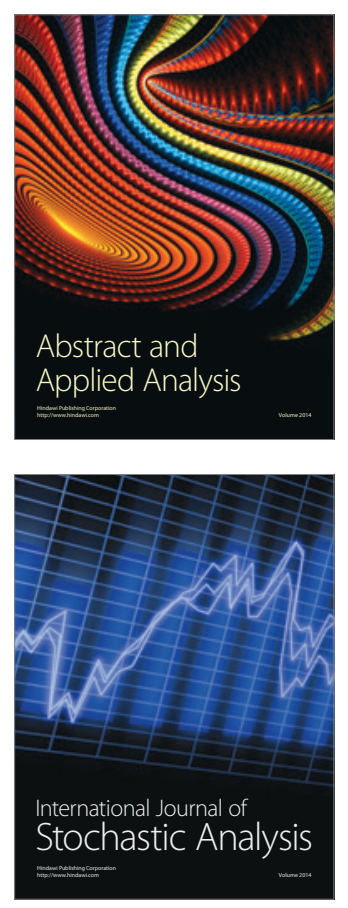

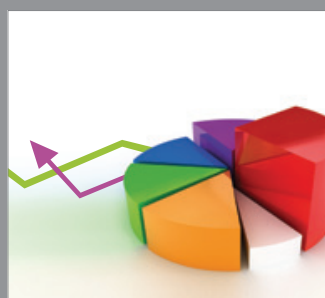

ournal of

Probability and Statistics

Promensencen
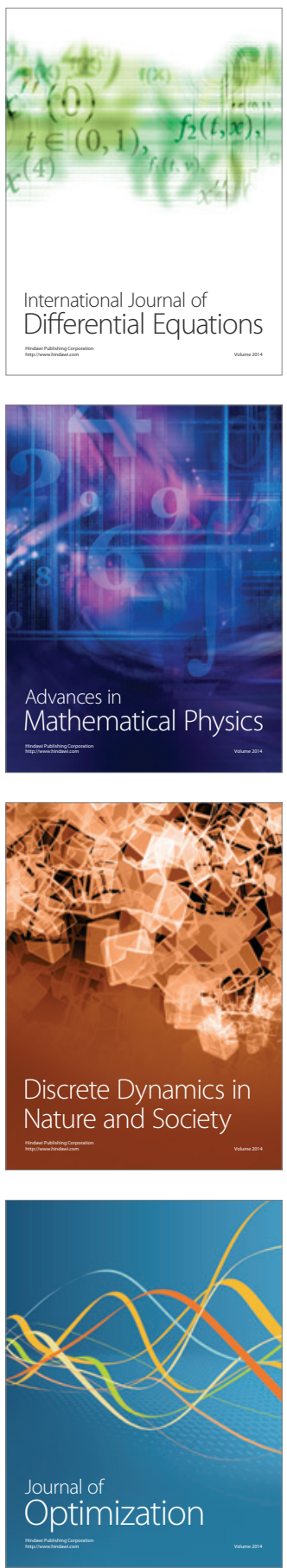